\title{
The Use of Endoscopic Clipping in Preventing Delayed Complications after Endoscopic Resection for Superficial Non-Ampullary Duodenal Tumors
}

\author{
Jee Young An', Byung-Wook Kim', Joon Sung Kim', Jae-Myung Park², Tae Ho Kim³ and Jaesin Lee ${ }^{1}$ \\ Department of Internal Medicine, ${ }^{1}$ Incheon St. Mary's Hospital, The Catholic University of Korea, Incheon, ${ }^{2}$ Seoul St. Mary's Hospital, \\ The Catholic University of Korea, Seoul, ${ }^{3}$ Bucheon St. Mary's Hospital, The Catholic University of Korea, Bucheon, Korea
}

Background/Aims: Endoscopic resection (ER) has recently been accepted as the standard treatment modality for superficial nonampullary duodenal tumors (SNADTs). However, the procedure can cause adverse events such as perforation and bleeding. This study aimed to investigate the efficacy of prophylactic clipping in the prevention of delayed complications.

Methods: A retrospective review of the medical records of patients who underwent ER for SNADT from 3 centers was performed. Patients were divided into 2 groups: the immediate clipping group (ICG) and the no clipping group (NCG). Various baseline characteristics and factors associated with the appearance of delayed complications, such as size of the lesion, tumor location, histologic type, and co-morbidities, were compared between the two groups.

Results: A total of 99 lesions from 99 patients were included in this study. Fifty-two patients were allocated into ICG and 47 patients were allocated into NCG. Delayed bleeding occurred in 1 patient from ICG and in 8 patients from NCG. Delayed perforation occurred in 1 patient from ICG and in 3 patients from NCG. There were no procedure-related deaths in both groups.

Conclusions: Although the use of endoscopic clipping seemed to reduce the risk of developing delayed complications, further studies using a prospective design is required. Clin Endosc 2021;54:563-569

Key Words: Clipping; Complication; Endoscopic resection; Non-ampullary duodenal tumor

\section{INTRODUCTION}

Superficial non-ampullary duodenal tumors (SNADTs) are defined as adenomas or adenocarcinomas in the duodenum that did not originate from the ampulla of Vater and are limited to the mucosal/submucosal layer. ${ }^{1,2}$ The incidence of SNADT among patients who underwent duodenoscopy is $0.03 \%$ to $0.4 \% .^{3}$ Although primary duodenal carcinomas are

Received: April 22, 2020 Revised: June 8, 2020

Accepted: June 17, 2020

Correspondence: Byung-Wook Kim

Division of Gastroenterology, Department of Internal Medicine, Incheon St. Mary's Hospital, The Catholic University of Korea, 56 Dongsu-ro, Bupyeong-gu, Incheon 21431, Korea

Tel: +82-32-280-5052, Fax: +82-32-280-5987, E-mail: gastro@catholic.ac.kr ORCID: https://orcid.org/0000-0002-2290-4954

(cc) This is an Open Access article distributed under the terms of the Creative Commons Attribution Non-Commercial License (http://creativecommons.org/ licenses/by-nc/3.0) which permits unrestricted non-commercial use, distribution, and reproduction in any medium, provided the original work is properly cited. rare, the prognosis of advanced duodenal carcinomas is very poor. ${ }^{4}$ Thus, early detection and treatment is crucial. Although the standard therapy for SNADT has yet to be established, endoscopic resection (ER) has shown to be beneficial for patients with SNADT. ${ }^{5-8}$

When mucosal defects occur after ER, pancreatic juice and bile acid may act as offensive agents and cause complications like delayed bleeding and delayed perforation. ${ }^{9,10}$ According to a previous report, the incidence of delayed perforations after ER for SNADT is 6.3\%. ${ }^{11}$ To prevent these complications, prophylactic procedures, such application of glycolic acid sheets, coagulation of visible vessels, and endoscopic clipping, have been introduced. ${ }^{12-18}$ However, previous reports on these procedures had several limitations: most of the studies were performed in single centers and heterogenous disease entities were included. Because of this, the prophylactic effects of endoscopic clipping have not been well-established. This study aimed to investigate the effects of prophylactic clipping 
immediately after ER for SNADT in the prevention of delayed complications.

\section{MATERIALS AND METHODS}

\section{Study design}

This study was a multicenter retrospective cohort study. Medical records dating from January 2007 to April 2018 from Incheon St. Mary's Hospital, Seoul St. Mary's Hospital, and Bucheon St. Mary's Hospital, The Catholic University of Korea were reviewed. Approval for the study was obtained from The Institutional Review Board of The Catholic University of Korea.

\section{Inclusion and exclusion criteria}

SNADTs that were removed endoscopically during this period were included in the study. Ampullary duodenal tumors and SNADTs other than adenomas and adenocarcinomas were excluded. Furthermore, patients who underwent additional endoscopic and/or surgical procedures for the management of complications, such as bleeding or perforation, during and/or immediately after the procedure were also excluded. In order to compare the data regarding these complications, patients were divided into two: the immediate clipping group (ICG) and the no clipping group (NCG).

\section{Endoscopic mucosal resection and endoscopic submucosal dissection procedures}

Endoscopic mucosal resection (EMR) and endoscopic submucosal dissection (ESD) were performed using either twin-channel multi-bending gastroscopes (GIF-2TQ260M; Olympus, Tokyo, Japan) or conventional gastroscopes (GIF-H260 or GIF-HQ290; Olympus) along with a $\mathrm{CO}_{2}$ insufflation system under conscious sedation. For EMR, a mixture of normal saline and diluted epinephrine (1:200,000) was injected into the submucosal layer. The lesions were then removed using snares and an electrosurgical unit (VIO300D, Endocut Q mode, effect 2; ERBE, Tübingen, Germany). After removal of the lesion, proton pump inhibitors (PPIs) were administered intravenously for 2 days followed by oral administration for 4 weeks.

For ESD, chromoendoscopy was performed using acetic acid (1.5\%) and indigo carmine (0.2\%). Dot markings were made $5 \mathrm{~mm}$ from the tumor margin using argon plasma coagulation. The same mixture used in EMR was also injected into the submucosal layer. Afterwards, an initial incision was made outside the marks using either a dual knife (KD-655; Olympus) or a hook knife (KD-620LR; Olympus). A knife was then inserted into the initial incision and an electrical current was applied using an electrosurgical unit (VIO300D, Endocut I mode, effect 2; ERBE) to complete a circumferential mucosal incision around the lesion. The submucosal layer was then dissected using either a dual knife or a hook knife. After removal, PPIs were administered in the same way described in the EMR procedure.

\section{Clipping procedures}

Whether clipping was used to close the defect was decided by the endoscopists' preferences. After ER, the mucosal defect was closed using clips (HX-610-090L; Olympus Medical Systems Co., Tokyo, Japan) delivered by a clip-fixing device (HX110UR; Olympus Medical Systems). Complete closure was defined as an invisible mucosal defect after clipping.

\section{Primary and secondary outcomes}

Delayed complications included delayed bleeding and delayed perforations; the incidence rates of these complications were considered as the primary outcomes. Delayed bleeding was defined as the presence of melena and/or hematemesis occurring at least 24 hours after procedure, or by duodenoscopic documentation of visible hemorrhages from the mucosal defect that eventually required endoscopic intervention. Delayed perforation was defined as the presence of intra-peritoneal free air on plain chest X-ray and/or on abdominal computed tomography at least 24 hours after the procedure. Factors associated with delayed complications, such as age, sex, comorbidity, anticoagulant use, and tumor characteristics (tumor location, tumor size, and tumor pathology), along with the treatment modalities used (EMR vs. ESD) were then analyzed. Secondary outcomes, which include the duration of hospitalization, transfusion requirements, the need for surgery, and local recurrences, were compared between ICG and NCG.

\section{Statistical analysis}

Dependent variables were determined using descriptive data. Continuous data were expressed as means \pm standard deviation. The statistical differences between the baseline characteristics of patients from ICG and NCG were assessed using the student $t$-test for continuous variables, and the chi-square test or the Fisher exact test for categorical variables. Factors associated with the occurrence of delayed complications were also analyzed. SPSS version 13.0 (SPSS Inc., Chicago, IL, USA) was used for statistical analyses.

\section{RESULTS}

\section{Baseline characteristics}

ER was performed by a total of 20 endoscopists with more 
than 3 years of clinical experience each. A total of 270 patients underwent ER (EMR or ESD) for SNADT from January 2007 to April 2018. Forty six patients with Brunner's gland hyperplasia, 36 patients with neuroendocrine tumors, 25 patients with inflammatory polyps, 24 patients with hyperplastic polyps, 12 patients with hamartomatous polyps, 5 patients with lipoma, 4 patients with heterotopic gastric mucosa, 3 patients with Brunner's gland adenoma, 3 patients with pyloric gland adenoma, 2 patients with ectopic pancreas, 2 patients with ganglioneuroma, 1 patient with leiomyoma, 1 patient with duodenal cyst, and 1 patient with gastrointestinal stromal tumor were excluded. Additionally, 1 patient that developed perforation during the procedure, and 5 patients that experienced hemorrhage immediately after the procedure, were also excluded. Because Brunner's glands originate from the submucosal layer, and that pyloric gland adenomas differ in nature from adenomas and adenocarcinomas of the duodenum, patients with these conditions were excluded. After the process of exclusion, 99 of the 270 original patients were analyzed in this study (Fig. 1). There were no differences in baseline characteristics such as age, male to female ratio, presence of comorbidities, social histories such as alcohol consumption and smoking, and current drug histories between the 2 groups (Table 1). An example of endoscopic clipping after EMR is presented in Fig. 2.

Complete closure was attained in every case. However, closure was not successful in 1 case where immediate perforation occurred and in 1 case where bleeding occurred immediately after closure (Fig. 1). Endoscopic clipping was more commonly performed on lesions located in the 2nd portion of the duodenum ( $p=0.01$; Table 2 ) and on adenomas presenting with low grade dysplasia histologically ( $p=0.01$; Table 2 ). There were no differences in the removal technique used, tumor size, circumferential location, histologic type, and the type of resection performed, whether en bloc resection or complete, between the 2 groups (Table 2).

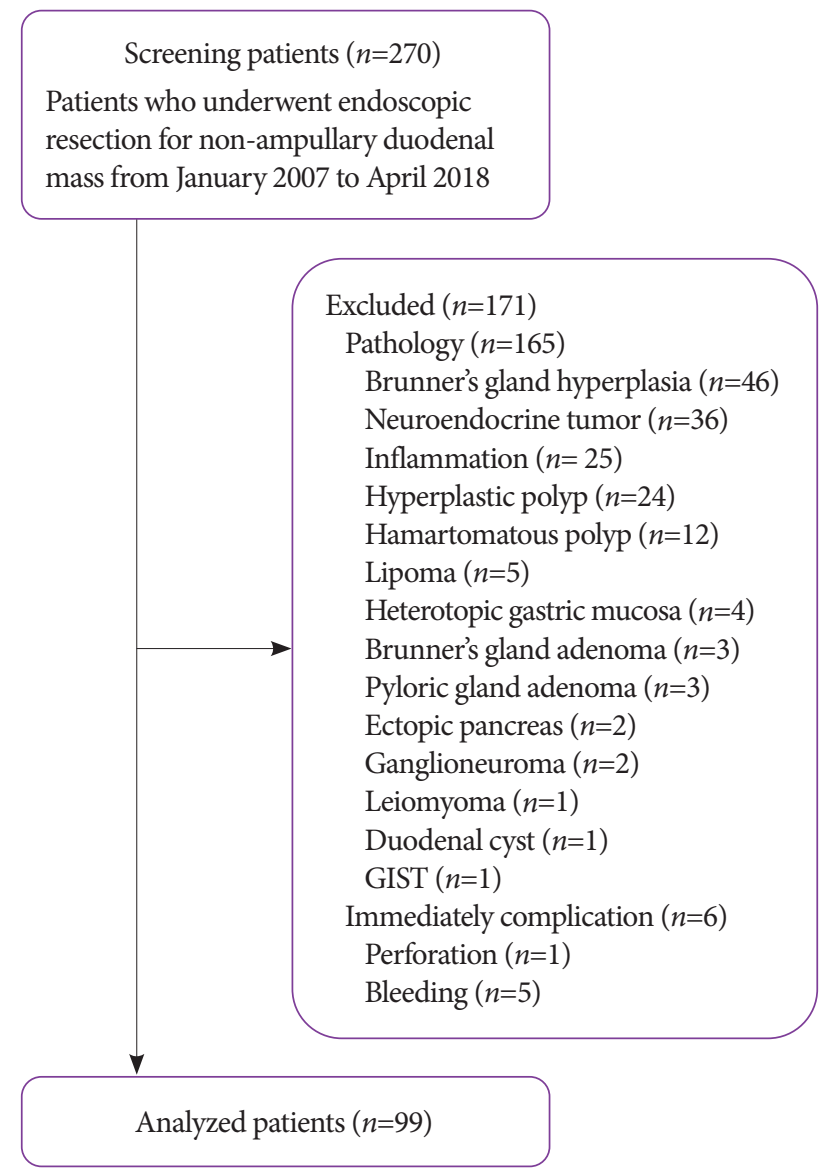

Fig. 1. Flow diagram of this study. GIST, gastrointestinal stromal tumor.

Table 1. Baseline Characteristics of the Immediate Clipping Group and the No Clipping Group

\begin{tabular}{lccc}
\hline & ICG $(\boldsymbol{n = 5 2})$ & NCG $(\boldsymbol{n}=\mathbf{4 7})$ & $\boldsymbol{p}$-value \\
\hline Age, yr (mean \pm SD) & $53.1 \pm 10.9$ & $54.1 \pm 14$ & 0.73 \\
Male:Female & $36: 16$ & $29: 18$ & 0.43 \\
Co-morbidities & & & \\
Hypertension & $9(17.3 \%)$ & $13(27.7 \%)$ & 0.22 \\
Diabetes & $7(13.5 \%)$ & $5(10.6 \%)$ & 0.67 \\
Other comorbidities & $16(30.8 \%)$ & $14(29.8 \%)$ & 0.91 \\
Smoking & $8(15.4 \%)$ & $7(14.9 \%)$ & 0.95 \\
Alcohol & $14(26.9 \%)$ & $11(23.4 \%)$ & 0.69 \\
Anticoagulant or antiplatelet agents & $5(9.6 \%)$ & $4(8.5 \%)$ & 1.00 \\
\hline
\end{tabular}

ICG, immediate clipping group; NCG, no clipping group; SD, standard deviation. 

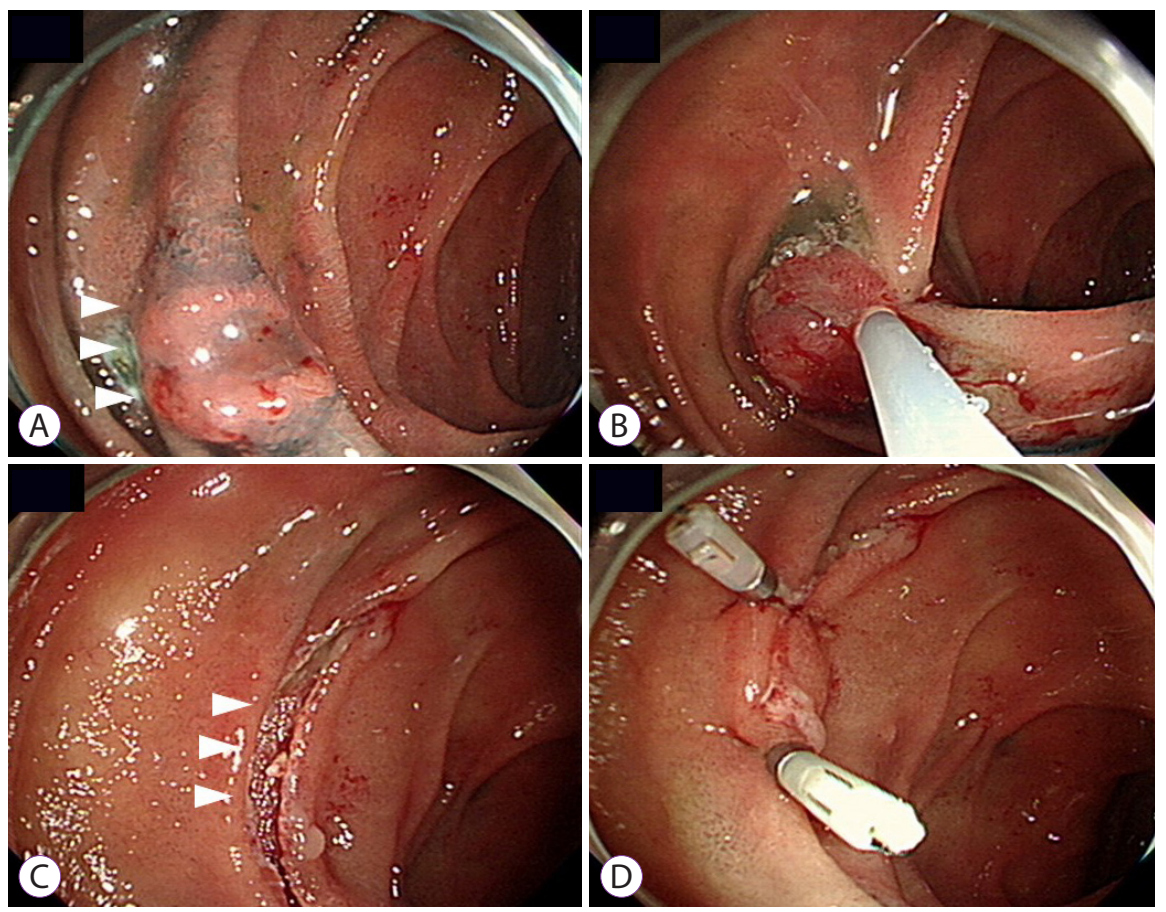

Fig. 2. Endoscopic clipping after endoscopic mucosal resection for duodenal adenoma. (A) An adenoma is found at the second portion of the duodenum (white arrow heads). (B) Endoscopic mucosal resection with snare is performed. (C) A mucosal defect is noted after endoscopic mucosal resection (white arrow heads). (D) The mucosal defect is closed using an endoscopic clipping device.

Table 2. Endoscopic Characteristics of Duodenal Lesions in the Immediate Clipping Group and the No Clipping Group

\begin{tabular}{|c|c|c|c|}
\hline & ICG $(n=52)$ & NCG $(n=47)$ & $p$-value \\
\hline Removal technique & & & 1.00 \\
\hline EMR & $49(94.2 \%)$ & $44(93.6 \%)$ & \\
\hline ESD & $3(5.8 \%)$ & $3(6.4 \%)$ & \\
\hline Tumor size $(\mathrm{cm})^{\mathrm{a})}$ & 1.0 (range, $0.3-3.5$ ) & 1.0 (range, $0.5-3.0$ ) & 1.00 \\
\hline Location & & & 0.01 \\
\hline 1st portion & $9(17.3 \%)$ & $20(42.6 \%)$ & \\
\hline 2nd portion & $43(82.7 \%)$ & $27(57.4 \%)$ & \\
\hline Circumferential location & & & 0.14 \\
\hline Anterior wall & $16(30.8 \%)$ & $16(34.0 \%)$ & \\
\hline Lateral wall & $11(21.2 \%)$ & $3(6.4 \%)$ & \\
\hline Posterior wall & $11(21.2 \%)$ & $16(34.0 \%)$ & \\
\hline Medial wall & $14(26.9 \%)$ & $12(25.5 \%)$ & \\
\hline Final histologic diagnosis & & & 0.01 \\
\hline Adenocarcinoma & $1(1.9 \%)$ & $3(6.4 \%)$ & \\
\hline HGD & $3(5.8 \%)$ & $11(23.4 \%)$ & \\
\hline LGD & $48(92.3 \%)$ & $33(70.2 \%)$ & \\
\hline Endoscopic findings & & & 0.41 \\
\hline Elevated & $21(40.4 \%)$ & $23(48.9 \%)$ & \\
\hline Flat & $21(40.4 \%)$ & $13(27.7 \%)$ & \\
\hline Depressed & $10(19.2 \%)$ & $11(23.4 \%)$ & \\
\hline En bloc resection & $42(80.8 \%)$ & $40(85.1 \%)$ & 0.57 \\
\hline Complete resection & $37(71.2 \%)$ & $33(70.2 \%)$ & 0.27 \\
\hline
\end{tabular}

EMR, endoscopic mucosal resection; ESD, endoscopic submucosal dissection; HGD, high grade dysplasia; ICG, immediate clipping group; LGD, low grade dysplasia; NCG, no clipping group.

${ }^{\text {a)}}$ Long diameter of the lesion. 


\section{Primary and secondary outcomes}

Among the 99 patients who underwent ER, delayed complications occurred in 13 patients (13.1\%). Delayed bleeding occurred in 9 patients $(9.1 \%)$ and delayed perforation occurred in 4 patients (4.0\%). Delayed bleeding occurred in 1 patient from ICG and in 8 patients from NCG (1.9\% vs. $17.0 \%$, $p=0.01$; Table 3). However, delayed perforation occurred in 1 patient from ICG and in 3 patients from NCG (1.9\% vs. $6.4 \%$, $p=0.34$; Table 3). Patients from ICG showed a tendency to have shorter lengths of hospital stay than those from NCG

Table 3. Clinical Outcomes of the Immediate Clipping Group and the No Clipping Group

\begin{tabular}{lccc}
\hline Variables & ICG $(\boldsymbol{n}=\mathbf{5 2})$ & NCG $(\boldsymbol{n}=\mathbf{4 7})$ & $\boldsymbol{p}$-value \\
\hline Hospitalization days (median, range) & 3 (range, $1-18)$ & 4 (range, $1-17)$ & 0.06 \\
Transfusion & $1(1.9 \%)$ & $5(10.6 \%)$ & 0.10 \\
Complications & $2(3.8 \%)$ & $11(23.4 \%)$ & 0.01 \\
Delayed perforation & $1(1.9 \%)$ & $3(6.4 \%)$ & 0.34 \\
Delayed bleeding & $1(1.9 \%)$ & $8(17.0 \%)$ & 0.01 \\
Surgery & $1(1.9 \%)$ & $3(6.4 \%)$ & 0.34 \\
\hline
\end{tabular}

ICG, immediate clipping group; NCG, no clipping group.

Table 4. Factors Associated with Delayed Complications

\begin{tabular}{|c|c|c|c|}
\hline Variables & $\begin{array}{l}\text { With delayed complications } \\
\qquad(n=13)\end{array}$ & $\begin{array}{l}\text { Without delayed complications } \\
\qquad(n=86)\end{array}$ & $p$-value \\
\hline Age (yr) & $52.1 \pm 11.4$ & $53.8 \pm 12.6$ & 0.628 \\
\hline Male & $9(69.2 \%)$ & $56(65.1 \%)$ & 1.000 \\
\hline Comorbidities & $2(15.4 \%)$ & $28(32.6 \%)$ & 0.333 \\
\hline Smoking & $1(7.7 \%)$ & $14(16.3 \%)$ & 0.685 \\
\hline Alcohol & $4(30.8 \%)$ & $21(24.4 \%)$ & 0.733 \\
\hline Anticoagulant and/or antiplatelet agent & $1(7.7 \%)$ & $8(9.3 \%)$ & 0.333 \\
\hline Endoscopic resection technique & & & 0.029 \\
\hline EMR & $10(76.9 \%)$ & $83(96.5 \%)$ & \\
\hline ESD & $3(23.1 \%)$ & $3(3.5 \%)$ & \\
\hline Endoscopic closure & $2(15.4 \%)$ & $50(58.1 \%)$ & 0.004 \\
\hline Mean tumor size (long axis, $\mathrm{cm}$ ) & $1.32 \pm 0.56$ & $1.17 \pm 0.72$ & 0.251 \\
\hline Tumor location & & & 0.516 \\
\hline 1st portion & $5(38.5 \%)$ & $24(27.9 \%)$ & \\
\hline 2nd portion & $8(61.5 \%)$ & $62(72.1 \%)$ & \\
\hline Histopathology & & & 0.953 \\
\hline Adenocarcinoma & $0(0.0 \%)$ & $4(4.7 \%)$ & \\
\hline HGD & $6(46.2 \%)$ & $8(9.3 \%)$ & \\
\hline LGD & $7(53.8 \%)$ & $74(86.0 \%)$ & \\
\hline Macroscopic findings & & & 0.316 \\
\hline Elevated & $5(38.5 \%)$ & $39(45.3 \%)$ & \\
\hline Flat & $3(23.1 \%)$ & $31(36.0 \%)$ & \\
\hline Depressed & $5(38.5 \%)$ & $16(18.6 \%)$ & \\
\hline En bloc resection & $12(92.3 \%)$ & $70(81.4 \%)$ & 0.457 \\
\hline Complete resection & $8(61.5 \%)$ & $68(79.1 \%)$ & 0.173 \\
\hline
\end{tabular}

EMR, endoscopic mucosal resection; ESD, endoscopic submucosal dissection; HGD, high grade dysplasia; LGD, low grade dysplasia. 
Table 5. Multivariate Analysis of the Factors Associated with Delayed Complications

\begin{tabular}{lcc}
\hline Variables & Odds ratio $(\mathbf{9 5 \%}$ confidence interval) & $\boldsymbol{p}$-value \\
\hline Endoscopic resection technique & $13.0(1.7-102.1)$ & 0.025 \\
Endoscopic closure & $8.7(1.6-48.7)$ & 0.014 \\
\hline
\end{tabular}

(3.6 \pm 2.5 vs. $4.7 \pm 3.3, p=0.06$; Table 3$)$. There were no differences in the need for transfusion and in the need for surgery between the 2 groups (Table 3 ).

\section{Factors associated with delayed complications}

Univariate analysis showed that the delayed complications experienced by the patients were related to ER methods, endoscopic clipping, and histologic tumor types (Table 4). In multivariate analysis, ER methods (odds ratio [OR], 13.0; 95\% confidence interval $[\mathrm{CI}], 1.7$ to $102.1 ; p=0.025)$ and endoscopic clipping (OR, 8.7; 95\% CI, 1.6 to $48.7 ; p=0.014$ ) were independently associated with the occurrence of delayed complications (Table 5).

\section{Management of patients with delayed complications}

All 9 patients with delayed bleeding were managed endoscopically and all 4 patients with delayed perforation were managed by laparoscopic simple closure successfully. There were no deaths among patients with delayed complications and the longest length of hospitalization was 19 days.

\section{DISCUSSION}

The strength of this study lies in the fact that it was conducted as a multi-center study with 20 endoscopists performing the procedures. The results of studies from single centers with a limited number of endoscopists are difficult to generalize. In this study, endoscopic closure was performed with clips alone and without the concurrent use of endoloops as was done in the previous study. ${ }^{10}$ The lesions in this study were also pathologically homogenous compared to previous reports, which included various submucosal lesions. ${ }^{17}$ The outcomes of mucosal closure after ER between lesions originating from the submucosal and mucosal layers may differ since the depth of the mucosal defect after ER is different.

In this study, we found that the type of ER performed and the use of endoscopic clipping were associated with delayed complications, especially delayed bleeding after ER for SNADT. Considering that EMR cannot replace ESD, endoscopic clipping may reduce the incidence of delayed complications. Although delayed perforation was not related to endoscopic clipping, it occurred in only 4 cases among 99 patients and thus a conclusion cannot be made from this study. Further prospective studies done at larger scales are anticipated to help determine the presence of an association.

Delayed complications were not associated with the administration of anti-thrombotic agents. It is possible that most physicians were very cautious with re-starting anti-thrombotic agents due to concerns about the possibility of causing delayed bleeding after ER for SNADT. Another possible explanation is that the co-administration of PPIs and mucoprotective agents helped in reducing the risk of developing delayed bleeding. ${ }^{19}$ The size of the lesion was not associated with delayed complications as well. However, although not intended, the lesions included in this study was relatively small. The median largest diameter of the lesions included in this study was $1.0 \mathrm{~cm}$ for both ICG (range, 0.3-3.5 cm) and NCG (range, 0.5-3.0 cm); this is in contrast to the value of $2.3 \mathrm{~cm}$ in the previous study. ${ }^{10}$

The duodenum has unique anatomical features-it is narrow and crooked, making it difficult to maintain the visual field during ER. ${ }^{9}$ Additionally, the duodenum has abundant blood vessels in the submucosal layer which causes more instances of delayed bleeding than any other part of gastrointestinal tract. ${ }^{12,13}$ When delayed complications cannot be managed by endoscopic procedures, the use of aggressive surgical treatments, such as prancreaticojejunostomy, is inevitable. Therefore, numerous prophylactic measures to reduce these complications have been introduced. One study showed that the application of over-the scope clippings may reduce the risk of severe adverse events after duodenal ESD. ${ }^{14}$ Another study insisted that the use of polyglycolic acid sheets and fibrin glue may prevent the occurrence of delayed perforation after duodenal ER. ${ }^{15}$ There was another study that reported that performing endoscopic closure using clips and an endoloop was associated with reduced risks for the development of delayed bleeding after duodenal ESD. ${ }^{10}$ Successful suturing with clips and strings after duodenal ER has been introduced. ${ }^{16}$ Compared to these new methods, endoscopic clipping is relatively simple, cheap, and easy to access. ${ }^{20-22}$

Despite these advantages, endoscopic clipping has some limitations. In cases of failure, endoscopic clipping might cause perforation or bleeding since the duodenal wall is relatively thin. ${ }^{15,17}$ In addition, complete closure of a large mucosal defect after ER for SNADT is sometimes difficult. ${ }^{14,16}$ Fortunately in this study, endoscopic clipping was successfully performed 
without complications in every case; this was mainly due to relatively small size of the lesions in this study. The longest diameter of the biggest lesion in this study was $3.5 \mathrm{~cm}$.

There are some limitations in this study. First, due to its retrospective design, selection bias may exist. Second, although not intended, relatively large lesions were not included in this study. Third, although we reviewed medical records spanning over a decade in 3 centers, ER for SNADT was performed in only 270 patients which is a relatively small sample size. Fourth, there is a possibility that failed endoscopic clipping might not be described in medical records and thus the success rate of the procedure could not be calculated.

In conclusion, endoscopic clipping might reduce the risk of delayed complications after ER for SNADT. Further prospective studies with larger scales are anticipated in the future.

\section{Conflicts of Interest}

The authors have no potential conflicts of interest.

\author{
Funding \\ None.

\section{Author Contributions} \\ Conceptualization: Byung-Wook Kim \\ Data curation: Jee Young An, Joon Sung Kim \\ Formal analysis: JYA, BWK \\ Investigation: JYA, BWK, JSK, Jae-Myung Park, Tae Ho Kim, Jaesin Lee \\ Methodology: JYA, BWK, JSK \\ Project administration: BWK \\ Resources: JYA, JMP, THK, JL \\ Software: JYA, JSK \\ Supervision: BWK \\ Validation: BWK \\ Visualization: JYA \\ Writing-original draft: JYA \\ Writing-review\&editing: BWK, JSK, JMP, THK, JL
}

\section{ORCID}

Jee Young An Byung-Wook Kim: Joon Sung Kim: Jae-Myung Park: Tae Ho Kim: Jaesin Lee: https://orcid.org/0000-0002-4578-0712
https://orcid.org/0000-0002-2290-4954
https://orcid.org/0000-0001-9158-1012
https://orcid.org/0000-0002-1534-7467
https://orcid.org/0000-0003-2015-5176
https://orcid.org/0000-0001-8981-0445

\section{REFERENCES}

1. Fu YW, Lin MS, Yang B, Yu H, Jiao X, Huang JX. An early superficial non-ampullary duodenal tumor cured with endoscopic submucosal dissection: a case report. Oncol Lett 2017;14:3755-3759.

2. Klein A, Nayyar D, Bahin FF, et al. Endoscopic mucosal resection of large and giant lateral spreading lesions of the duodenum: success, adverse events, and long-term outcomes. Gastrointest Endosc 2016;84:688-
696.

3. Shibagaki K, Ishimura N, Kinoshita Y. Endoscopic submucosal dissection for duodenal tumors. Ann Transl Med 2017;5:188.

4. Cortese AF, Cornell GN. Carcinoma of the duodenum. Cancer 1972;29:1010-1015.

5. Kakushima N, Kanemoto H, Tanaka M, Takizawa K, Ono H. Treatment for superficial non-ampullary duodenal epithelial tumors. World J Gastroenterol 2014;20:12501-12508.

6. Valerii G, Tringali A, Landi R, et al. Endoscopic mucosal resection of non-ampullary sporadic duodenal adenomas: a retrospective analysis with long-term follow-up. Scand J Gastroenterol 2018;53:490-494.

7. Pérez-Cuadrado-Robles E, Quénéhervé L, Margos W, et al. Comparative analysis of ESD versus EMR in a large European series of non-ampullary superficial duodenal tumors. Endosc Int Open 2018;6:E1008-E1014.

8. Pérez-Cuadrado-Robles E, Quénéhervé L, Margos W, et al. ESD versus EMR in non-ampullary superficial duodenal tumors: a systematic review and meta-analysis. Endosc Int Open 2018;6:E998-E1007.

9. Inoue $\mathrm{T}$, Uedo $\mathrm{N}$, Yamashina $\mathrm{T}$, et al. Delayed perforation: a hazardous complication of endoscopic resection for non-ampullary duodenal neoplasm. Dig Endosc 2014;26:220-227.

10. Hoteya S, Kaise M, Iizuka T, et al. Delayed bleeding after endoscopic submucosal dissection for non-ampullary superficial duodenal neoplasias might be prevented by prophylactic endoscopic closure: analysis of risk factors. Dig Endosc 2015;27:323-330.

11. Fanning SB, Bourke MJ, Williams SJ, Chung A, Kariyawasam VC. Giant laterally spreading tumors of the duodenum: endoscopic resection outcomes, limitations, and caveats. Gastrointest Endosc 2012;75:805-812.

12. Hoteya S, Furuhata T, Takahito T, et al. Endoscopic submucosal dissection and endoscopic mucosal resection for non-ampullary superficial duodenal tumor. Digestion 2017;95:36-42.

13. Fujihara S, Mori H, Kobara H, et al. Management of a large mucosal defect after duodenal endoscopic resection. World J Gastroenterol 2016;22:6595-6609.

14. Tashima T, Ohata K, Sakai E, et al. Efficacy of an over-the-scope clip for preventing adverse events after duodenal endoscopic submucosal dissection: a prospective interventional study. Endoscopy 2018;50:487-496.

15. Doyama H, Tominaga K, Yoshida N, Takemura K, Yamada S. Endoscopic tissue shielding with polyglycolic acid sheets, fibrin glue and clips to prevent delayed perforation after duodenal endoscopic resection. Dig Endosc 2014;26(Suppl 2):41-45.

16. Yahagi N, Kato M, Ochiai Y, et al. Outcomes of endoscopic resection for superficial duodenal epithelial neoplasia. Gastrointest Endosc 2018;88:676-682.

17. Ye LP, Mao XL, Zheng HH, et al. Safety of endoscopic resection for duodenal subepithelial lesions with wound closure using clips and an endoloop: an analysis of 68 cases. Surg Endosc 2017;31:1070-1077.

18. Kato M, Ochiai Y, Fukuhara S, et al. Clinical impact of closure of the mucosal defect after duodenal endoscopic submucosal dissection. Gastrointest Endosc 2019;89:87-93.

19. Fujiwara S, Morita Y, Toyonaga T, et al. A randomized controlled trial of rebamipide plus rabeprazole for the healing of artificial ulcers after endoscopic submucosal dissection. J Gastroenterol 2011;46:595-602.

20. Nishizawa T, Suzuki H, Goto O, Ogata H, Kanai T, Yahagi N. Effect of prophylactic clipping in colorectal endoscopic resection: a meta-analysis of randomized controlled studies. United European Gastroenterol J 2017;5:859-867.

21. Feagins LA, Nguyen AD, Iqbal R, Spechler SJ. The prophylactic placement of hemoclips to prevent delayed post-polypectomy bleeding: an unnecessary practice? A case control study. Dig Dis Sci 2014;59:823-828.

22. Shirai Y, Ohki T, Yamamoto M. Application of the clip method, using thread, for duodenal endoscopic mucosal resection. BMJ Case Rep 2016;2016:bcr2016215677. 